\title{
Methanogenic community composition in an organic waste mixture in an anaerobic bioreactor**
}

\author{
Agata Gryta ${ }^{1}$, Karolina Oszust ${ }^{1}$, Małgorzata Brzezińska ${ }^{1}$, Krzysztof Ziemiński ${ }^{2}$, Nina Bilińska-Wielgus ${ }^{1}$, \\ and Magdalena Frac ${ }^{1 *}$ \\ ${ }^{1}$ Institute of Agrophysics, Polish Academy of Sciences, Doświadczalna 4, 20-290 Lublin, Poland \\ ${ }^{2}$ Institute of Fermentation Technology and Microbiology, Łódź University of Technology, Wólczańska 171/173, 90-924 Łódź, Poland
}

Received October 25, 2016; accepted June 1, 2017

\begin{abstract}
A b s t r a c t. The aim of the study was to elucidate the substantial relationship between the compositions of methanogen community that assembles in the anaerobic digester mass and link it to methane production activity. The results of the metagenomic studies were used to evaluate how the methanogen structure changes during an anaerobic digestion process under various waste retention times $(21,23,25,29,33,39,47$ and 61 days). Phylogenetically coherent populations of methanogens were assessed by $16 \mathrm{~S}$ rRNA gene next-generation sequencing and terminal restriction fragment length polymorphism fingerprinting of a specific molecular marker, the mcrA gene. The results indicated multiple phylogenetically diverse methanogen populations associated with the various steps of anaerobic digestion. The stages of the anaerobic digestion process and waste retention times determine the microbial composition. The most dominant and acclimated microbial communities in all samples belonged to the genera Methanosaeta and Methanobacterium. The methane yield was consistent with the results of the microbial community structure, which indicated that acetotrophic Methanosaeta was the most active and most important during the methanogenic stage.

$\mathrm{K}$ e y w o r d s: anaerobic digestion, methanogenic activity, methanogenic Archaea, NGS, t-RFLP
\end{abstract}

\section{INTRODUCTION}

In recent years, the amount of agricultural wastes has increased rapidly. Dairy sewage sludge and fruit waste with high organic matter content (more than 60\%) can be used in anaerobic digestion as a major source of renewable energy (Frąc and Ziemiński, 2012). Methanogenesis is an essential biochemical process involved in the mineralization of organic matter in anaerobic conditions and in degradation of certain toxins (Whang et al., 2015). Anaerobic digestion

*Corresponding author e-mail: m.frac@ipan.lublin.pl

**This work was funded from the budget for science by the National Centre for Research and Development in Poland, LIDER Programme 2011-2014. is microbially mediated engineering with the goals of degradation and stabilisation of organic matter, which results in the production of energy-rich composites. This process involves four sequential steps: hydrolysis, fermentation (acidogenesis), acetogenesis (dehydrogenation) and methanogenesis (acetoclastic or hydrogenotrophic), all of which depend on the synergistic interactions of microorganisms that form a complex metabolic network. This special process is catalysed especially by methanogenic Archaea. This unrepeatable group of microorganisms has the capability to produce methane from basic substrates, such as $\mathrm{CO}_{2}, \mathrm{H}_{2}$, acetate or $\mathrm{C} 1$ compounds $e . g$. methanol, methylamines and methylthiols (Frąc and Ziemiński, 2012; Walter et al., 2012).

Methanogens reside in many anaerobic environments, such as sediments, digesters and municipal waste landfill sites (Garcia et al., 2000; Song et al., 2015), and are a difficult group to isolate or culture under laboratory conditions (Enitan et al., 2014; Pervin et al., 2013). Therefore, the development of molecular methods that are independent from cultivation is gaining importance. This approach allows the genetic diversity and dynamics of methanogenic Archaea to be analysed. Methanogens may be efficiently targeted in molecular ecological analyses by using a specific molecular marker, such as the mcr $A$ gene, which encodes the $\alpha$-subunit of methyl-coenzyme $M$ reductase (MCR) (EC 2.8.4.1). This is the key enzyme of methanogenesis, which reduces the coenzyme M-bound methyl group to methane (Shah et al., 2014). This enzyme is unique to methanogens, where the highly conserved gene $m c r A$ is found only in this group of microorganisms, whereas other enzymes involved in methanogenesis, such as methylene

(C) 2017 Institute of Agrophysics, Polish Academy of Sciences 
tetrahydromethanopterin dehydrogenase and methenyl tetrahydromethanopterin cyclohydrolase appear in another group of $\mathrm{C} 1$-utilising microorganisms. Therefore, identifying methanogens that occur in environmental samples by comparative $m c r A$ (as a functional marker) sequence analysis could be performed for the following methanogen groups: Methanosarcinaceae, Methanosaetaceae, Methanobacteriales, Methanococcales and Methanomicrobiales (Steinberg and Regan, 2008).

Terminal restriction fragment length polymorphism (t-RFLP) fingerprinting and next-generation sequencing (NGS) are methods frequently used in the methanogenic community analysis. T-RFLP analysis can be used to assess the genetic diversity, structure and dynamics of microbial populations (Kitts, 2001). T-RFLP is a PCR- (polymerase chain reactions) technique, where the amplicons are digested with restriction endonucleases. Subsequently, fluorescently labelled terminal restriction fragments (T-RFs) are separated and visualised by electrophoresis on an automated genetic analyser. Genetic analysis of community environmental samples by sequencing is also an important tool for understanding functional and ecological biodiversity. However, when a traditional DNA-sequencing method is used, each specimen must be analysed separately. This approach is inadequate for environmental samples, particularly for large-scale research. Environmental samples could contain mixtures of DNA from a large number of microorganisms. Obtaining DNA sequences from thousands of specimens present in an environmental sample requires the ability to read DNA from multiple levels in parallel; this is the primary advantage of next-generation sequencing (NGS) technologies.

The metagenomics workflow employs the 16S rRNA gene, which is commonly used for identification and classification of bacteria. This small-sized fragment (approximately $1500 \mathrm{bp}$ ) is ubiquitously present among prokaryotic organisms such as Bacteria and Archaea. In regular sequencing approaches, DNA is amplified with primers that match with conserved 16S rRNA regions. Amplicons include at least one hypervariable region that could be used to sequence and classify bacteria. The metagenomics workflow allows classification of reads at taxonomic levels: kingdom, phylum, class, order, family, and genus or species. This stage is based on comparison and matching of short sequences from the reads to a set of $16 \mathrm{~S}$ reference sequences bases. The total number of classified clusters for each sample at each taxonomic level is the analysis result.

There are a few scientific reports (Enitan et al., 2014; Esposito et al., 2012; Nikolausz et al., 2013) that have collated the microbial consortium involved in anaerobic digestion for precise characterisation and comparison to the methane yield efficiency derived from each stage consortium. We hypothesise that gaining a data set on the methanogen composition using metagenomic tools and comparing it with the potential effectiveness of their activi- ty will identify the essential step and methanogens involved in it, which will affect biogas profitability. This innovative approach was aimed to be achieved using knowledge of the community dynamics and the functional stability of the process under various retention times.

Despite an increasing number of biogas treatment plants that are being built in many countries, microbial biocenoses of biogas-producing facilities is still not fully understood and is often regarded as a black-box. Knowledge of microbial communities involved in anaerobic digestion processes is extremely important because the microorganism profile in a bioreactor depends on the type of biomass used (Abendroth et al., 2015).

The goal of this study is to investigate the effect of retention time on the methanogen community structures using the t-RFLP and NGS approaches and methanogenic activity. The objective of this study is also to provide a more precise view of the biological processes and primary microbial communities involved in anaerobic digestion of organic wastes.

\section{MATERIALS AND METHODS}

Total solid (TS), volatile solid (VS), ash, phosphorus, $\mathrm{pH}$ and volatile fatty acids were determined according to standard methods (APHA, 1998), total nitrogen (TKN) by Kjeldahl method and chemical oxygen demand (COD) by a modified Raposo method (Raposo et al., 2008).

A mixture of organic waste, which contained dairy sewage sludge, fruit waste, corn silage and grain decoction in proportions of $25,25,12$, and $38 \%$, respectively, was used in this study. The characteristics of the organic waste mixture used in the experiment are shown in Table 1. The anaerobic granular sludge harvested from an agricultural biogas plant was used as an inoculum in the anaerobic digestion process after concentrating by sedimentation (for $24 \mathrm{~h}$ ) using an Imhoff funnel.

T a b I e 1. Characteristics of organic waste mixture

\begin{tabular}{lc}
\hline Parameter & Mixture of waste \\
\hline Total solid (TS) (\%) & 11.70 \\
Volatile solid (VS) (\% TS) & 92.70 \\
Ash (\% TS) & 7.30 \\
Chemical oxygen demand (COD) & 1.45 \\
$\left.\mathrm{~g} \mathrm{O}_{2} \mathrm{~kg}^{-1} \mathrm{TS}\right)$ & \\
Total Kjeldahl nitrogen (TKN) (\% TS) & 4.90 \\
Phosphorus (g kg $\left.{ }^{-1} \mathrm{TS}\right)$ & 6.87 \\
$\mathrm{pH}$ & 4.36 \\
$\mathrm{C} / \mathrm{N}$ & 27.16 \\
\hline
\end{tabular}


The batch anaerobic digestion process was conducted with stirring (4 r.p.m.) under mesophilic conditions at a temperature of $37^{\circ} \mathrm{C}$ in glass chambers with a working volume of 21 . The operating anaerobic digestion temperature was maintained using a thermostat connected to a water jacket at the fermenter. Before anaerobic digestion, the $\mathrm{pH}$ of mixtures was adjusted to 7.0 using $\mathrm{Na}_{2} \mathrm{CO}_{3}$. To initiate the anaerobic digestion process, the inoculum described above was added to the reactor in the amount of $20 \%$ of the fermenter volume.

The retention times tested for the mixed waste were 21 , 23, 25, 29, 33, 39, 47 and 61 days (FM1, FM2, FM3, FM4, FM5, FM6, FM7 and FM8, respectively). The anaerobic digestion time was dependent on the retention time and included triple exchanges of the working volume fermenter. Each sample with various fermenter loads lasted until the working volume of the chamber was exchanged three times. After completion of the three exchanges of the fermenter working volume after each tested retention time, digested samples $(100 \mathrm{ml})$ were collected for metagenomic analysis. The volume of biogas produced was measured by an electronic flow rate-meter and the methane concentration in gaseous fermentation products was determined by GC, using an Agilent 7890A GC chromatograph (Ziemiński et al., 2014).

For the microbial structure analysis, sludge samples from the fermenter from the aforementioned stages were centrifuged at $4000 \mathrm{rcf}$ for $20 \mathrm{~min}$ at $4^{\circ} \mathrm{C}(5810 \mathrm{R}$, Eppendorf); the supernatants were removed and the sediments were stored at $4^{\circ} \mathrm{C}$ in a refrigerator. Another set of samples, FM1 to FM8 (collected to determine the methanogenic activity), was stored frozen $\left(-20^{\circ} \mathrm{C}\right)$ until processing.

The methanogenic activity, which is defined as the residual methane potential at certain stages of the anaerobic digestion process (FM1 to FM8), was determined based on methane production under controlled laboratory conditions. $0.5 \mathrm{~g}$ of each wet sample was weighed into $120 \mathrm{ml}$ glass serum bottles and suspended in $40 \mathrm{ml}$ of the inorganic medium containing per litre of distilled water as follows: $0.75 \mathrm{~g} \mathrm{KH}_{2} \mathrm{PO}_{4}, 1.45 \mathrm{~g} \mathrm{~K}_{2} \mathrm{HPO}_{4} 3 \mathrm{H}_{2} \mathrm{O}, 0.9 \mathrm{~g} \mathrm{NH}_{4} \mathrm{Cl}, 0.2 \mathrm{~g}$ $\mathrm{MgCl}_{2} \mathrm{H}_{2} \mathrm{O}, 0.5 \mathrm{~g} \mathrm{Na}_{2} \mathrm{~S} 9 \mathrm{H}_{2} \mathrm{O}, 9 \mathrm{ml}$ of microelements and $5 \mathrm{ml}$ of vitamins solutions at a $\mathrm{pH}$ of 7.0. The organic substrate available for methanogens was only the residual degradable organic substances still present in the tested samples during collection thereof.

In three replications, the bottles were sealed with rubber stoppers and aluminium cups and flushed for $3 \mathrm{~min}$ with a gas containing $\mathrm{N}_{2}, \mathrm{CO}_{2}$ and $\mathrm{H}_{2}(75: 10: 5 \mathrm{v} / \mathrm{v})$ to remove oxygen and create anaerobic conditions. Resazurin, which was added to the nutrient solution, allowed control of redox conditions (anaerobiosis) of the samples (Angelidaki and Sanders, 2004). Incubation was performed statically at a mesophillic temperature $\left(37^{\circ} \mathrm{C}\right)$. The experiment was continued for 35 days, until the daily methane production reached a maximum in all of the tested samples.
The concentration of methane in the headspace was measured with gas chromatographs Shimadzu GC-14B and GC-14A (Japan) equipped with a flame ionisation detector (FID) and a thermal conductivity detector (TCD) used for the detection of lower (initial $\mathrm{CH}_{4}$, in ppm) and higher $\left(\mathrm{CH}_{4}\right.$, in \%) concentrations, respectively. TCD operated with a $2 \mathrm{~m}$ column with a diameter of $3.2 \mathrm{~mm}$ packed with Porapak Q and with helium as a carrier gas flowing at a rate of $40 \mathrm{~cm}^{3} \mathrm{~min}^{-1}$. The temperature of the column and the detector was 40 and $60^{\circ} \mathrm{C}$, respectively. In the case of the FID detector, gases were separated on a column packed with a Porapak Q maintained at $80^{\circ} \mathrm{C}$, and the temperature of the injector was $150^{\circ} \mathrm{C}$; nitrogen was the carrier gas. The detector responses were calibrated using a certified gas standard (Air Products) containing $10 \mathrm{ppm}$ or $4 \% \mathrm{CH}_{4}$ in $\mathrm{He}$ (Lipiec et al., 2015). Gas samples $(0.2 \mathrm{ml})$ were taken through the septum with a pressure lock syringe and directly injected into the gas chromatograph. The gas pressure inside the vessel was measured using an Infield7C (UMS GmbH München) stitch-tensiometer equipped with a needle that allowed penetration into the vessel through the septum.

The corrected headspace of the bottles was calculated by subtracting the added amounts of the solution and a sample from the total bottle volume (Hansen et al., 2004). Correction for methane solubility in water was made using a published value of the Bunsen coefficient $(\alpha)$ of 0.026 at $37^{\circ} \mathrm{C}$. The volume of methane was corrected for standard temperature and pressure conditions using the ideal gas law and was obtained by multiplying the measured percentage of $\mathrm{CH}_{4}$ in the headspace (determined by $\mathrm{GC}$ analysis) by the corrected headspace volume. Methane production was presented as the cumulative values and expressed on an oven-dry weight basis $\left(105^{\circ} \mathrm{C}, 24 \mathrm{~h}\right)$, i.e. divided by the weight $(\mathrm{g})$ of the dry mass sample incubated in each bottle ( $\mathrm{ml} \mathrm{CH}_{4} \mathrm{~g}^{-1}$ d.m.). Methane production rates were calculated from the slope of the relationship $\left(\mathrm{R}^{2}>0.93\right)$ between the cumulative methane volume and incubation time (Kane et al., 2013). Moreover, daily changes in methane production were calculated by simply dividing the increase in the methane volume with time between adjacent measurements. The final methane amounts were also converted to moles $\mathrm{CH}_{4}$, with the assumption that a volume of $1 \mathrm{mmol}$ of $\mathrm{CH}_{4}$ at $37^{\circ} \mathrm{C}$ equals $25.4 \mathrm{ml}$.

The DNA from different sludge samples (FM1 to FM8) was extracted using a FASTDNA Spin Kit for Feces (MPBiomedicals) according to the protocol. DNA was eluted in $100 \mu 1$ of nuclease-free distilled water. The amount of DNA was determined by a spectrophotometer (NanoDrop ${ }^{\circledR}$ - 2000). The concentration of pure DNA was as follows: $111.67,24.2,32.17,39.77,38.50,22.77,68.84,27.80 \mathrm{ng} /$ $\mu 1$ for samples FM1, FM2, FM3, FM4, FM5, FM6, FM7 and FM8, respectively. The $m c r A$ gene was amplified via PCR using a pair of primers: the $m c r A$ - specific forward primer mlas (5'-GGT GGT GTM GGD TTC ACM CAR 
TA-3') and the reverse primer mcrA-rev (5'-CGT TCA TBG CGT AGT TVG GRT AGT-3') (Nikolausz et al., 2013). The reverse primer was 5'- labelled with the phosphoramidite fluorochrome 6-carboxyfluorescein (6-FAM). The PCR was performed in a total volume of 30 with $2 \mu 1$ of either isolated DNA and $15 \mu 1$ of RedTaq ReadyMix ${ }^{\mathrm{TM}}$ PCR Reaction Mix with $\mathrm{MgCl}_{2}$ (Sigma-Aldrich) with 0.06 $\mathrm{U} / \mu \mathrm{l}$ Taq DNA polymerase. The concentration of the primers used for the PCR was $0.5 \mu \mathrm{M}$.

The PCR reaction was performed in accordance to Steinberg and Regan (Steinberg and Regan, 2008), with the following temperature cycle: initial denaturation step at $95^{\circ} \mathrm{C}$ for $3 \mathrm{~min}$, followed by five cycles of denaturation at $95^{\circ} \mathrm{C}$ for $30 \mathrm{~s}$, annealing at $48^{\circ} \mathrm{C}$ for $45 \mathrm{~s}$ and extension at $72^{\circ} \mathrm{C}$ for $30 \mathrm{~s}$, with a ramp rate of $0.1^{\circ} \mathrm{C} \mathrm{s}^{-1}$ from the annealing to the extension temperature. These initial five cycles were followed by 30 cycles of denaturation at $95^{\circ} \mathrm{C}$ for $30 \mathrm{~s}$, annealing at $55^{\circ} \mathrm{C}$ for $45 \mathrm{~s}$, and extension at $72^{\circ} \mathrm{C}$ for $30 \mathrm{~s}$, followed by a final extension step at $72^{\circ} \mathrm{C}$ for $10 \mathrm{~min}$. The PCR products were separated on a $1.3 \%$ of agarose gel, which was stained with an ethidium bromide solution and visualised with UV excitation. The PCR products were purified using an ExoSAP-IT ${ }^{\circledR}$ PCR Products Purification Kit for $\mathrm{ABI}$ followed by incubation at $37^{\circ} \mathrm{C}$ for $15 \mathrm{~min}$ and then for $15 \mathrm{~min}$ at $80^{\circ} \mathrm{C}$. The purified amplification products $(2 \mu \mathrm{l})$ were digested in a $10 \mu \mathrm{l}$ reaction volume with $1 \mu \mathrm{l}$ of restriction endonucleases HaeIII (10U) and $1 \mu \mathrm{l}$ of buffer Tango with BSA. Restriction was performed at $37^{\circ} \mathrm{C}$ for $10 \mathrm{~h}$. Next, the reaction was halted by incubation at $80^{\circ} \mathrm{C}$ for $20 \mathrm{~min}$. Next, the digestion samples were precipitated with 0.1 volume of $3 \mathrm{M}$ sodium acetate $(\mathrm{pH} 5.5)$ and 2.5 volume of absolute ethanol. After washing in $70 \%$ ethanol, the DNA pellets were dried and suspended in $20 \mu \mathrm{l}$ of HiDi formamide containing 1.5\% ( $v / v)$ GeneScan- 600LIZ standard (Applied Biosystems). Fluorescently labelled terminal restriction fragments (T-RFs) were run through an ABI 3130 xl Genetic Analyser (Applied Biosystems) with NanoPOP ${ }^{\mathrm{TM}}$ Polymers (Nimagen), with two replicates for each restriction analysis to ensure reproducibility. T-RFLP data were analysed using GeneMapper v4.0 software (Applied Biosystems). Fluorescence signals of T-RFs in the size range of 50-500 bp were extracted to exclude potential primer peaks. Peaks with signals below 100 relative fluorescence units were also discarded from the analysis. Based on the presence, size and intensity of the peaks, methanogen genera were detected in the digested samples.

The MiSeq 2000 platform (Illumina Inc., San Diego, CA, USA) was applied to sequence the microorganism DNA isolated from digested samples from various stages of anaerobic digestion (FM 1 to FM8). The PCR reaction was performed with the primers 515F (5'-AAT GAT ACG GCG ACC ACC GAG ATC TAC ACT ATG GTA ATT GTG TGC CAG CMG CCG CGG TAA-3') and 806R (5'-CAA GCA GAA GAC GGC ATA CGA GAT XXX XXX XXX XXX AGT CAG TCA GCC GGA CTA CHV GGG TWT
CTA AT-3') for the 44 region of 16S rRNA (Caporaso et al., 2012). The PCR was made using a NebNext High-Fidelity 2xPCR Master Mix (New England BioLabs) according to the manufacturing protocol. The polymerase chain reaction conditions for the 16S rRNA genes were as follows: $94^{\circ} \mathrm{C}$ for $3 \mathrm{~min}, 35$ cycles of $94^{\circ} \mathrm{C} 45 \mathrm{~s}, 50^{\circ} \mathrm{C} 60 \mathrm{~s}$ and $72^{\circ} \mathrm{C} 90 \mathrm{~s}$ and a final extension at $72^{\circ} \mathrm{C}$ for $10 \mathrm{~min}$. The libraries were indexed in TrueSeq, Ilumina technology. Sequencing was performed in PE reads $2 \times 250$ bp with a v2 MiSeq regent kit (Illumina). Bioinformatics assay was based on the reference sequence database, Greengenes_13_05 (DeSantis et al., 2006) Bioinformatics analysis was performed using an algorithm from Qiime software. The analysis included the following steps: (1) demultiplexing of samples and adaptor cutting; (2) quality analysis and cluster analysis based on $97 \%$ of similarity using the uclust algorithm; (3) phylogenetic tree computation and taxonomic composition and diversity analysis. Sequences sharing identity greater than $97 \%$ identity were grouped into one operational taxonomic unit (OTU) using a distance-based OTU program. Application of MiSeq Reporter v2.3 allowed classifications at a species level. The taxonomy database for the metagenomics workflow was the Illumina version of the Greengenes database (DeSantis et al., 2006). The Illumina sequencing data were uploaded in the NCBI Sequence Read Archive database with the accession number SRP058331.

All statistical analyses were performed in Statistica software (version 10.0). One-way analysis of variance (LSD test) was used to test the differences in the methane production rate among the samples. A heatmap that shows relative abundances was generated based on the OTU table. Differences in the community composition were further explored using principle component analysis (PCA). The outcome of the PCA is derived by using uncorrelated principal components (PCs), which are linear combinations of variables that account for most of the variances within a data set.

\section{RESULTS AND DISCUSSION}

The cumulative methane production in the tested samples is shown in Fig. 1. Even after the first day of the incubation, small amounts of methane were observed in samples FM1 to FM4 (below 8 ppm in the headspace), although they were not observed in the other samples. After the three incubation days, methane was produced in all samples. Only low methane amounts were detected in samples FM7 and FM8 until the 7th day $\left(<0.75 \mathrm{ml} \mathrm{CH}_{4} \mathrm{~g}^{-1}\right.$ d.m.), whereas approximately $5 \mathrm{ml} \mathrm{CH}_{4} \mathrm{~g}^{-1} \mathrm{~d}$.m. was measured in samples FM1 to FM4. Sample FM8 exhibited the longest lag phase ( $>10$ days). Interestingly, the amount of methane reached approximately $72 \mathrm{ml} \mathrm{CH}_{4} \mathrm{~g}^{-1}$ d.m. after 14 days in samples FM2, FM3 and FM4 but only approximately 50 and $<5 \mathrm{ml} \mathrm{CH}_{4} \mathrm{~g}^{-1}$ d.m. in samples FM7 and FM8, respectively. Next, the dynamics of methane yield 


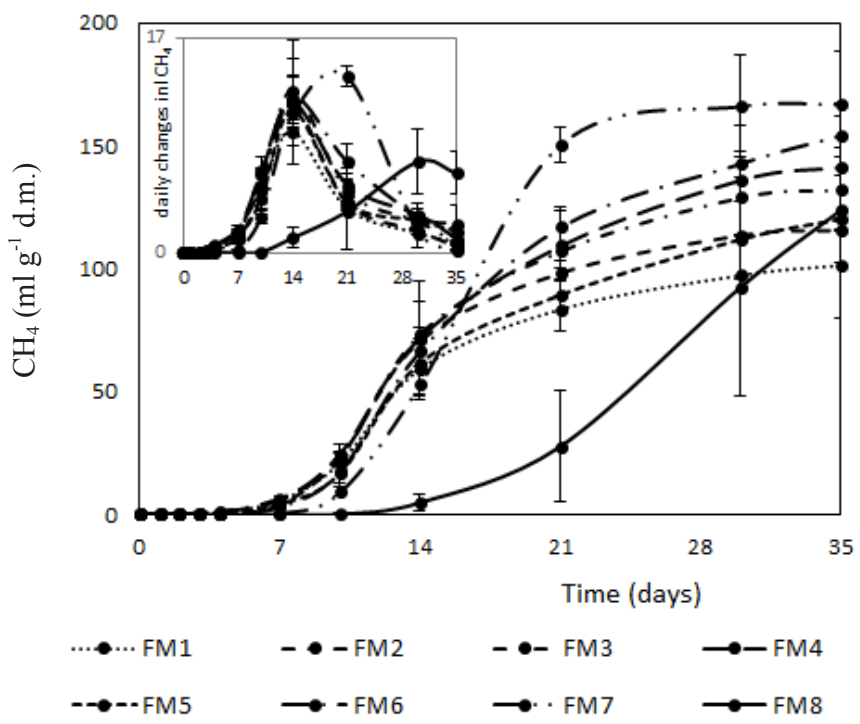

Fig. 1. Cumulative production of methane from tested samples in the function of time. The average of three replications $\pm \mathrm{SD}$, inset: daily changes in $\mathrm{CH}_{4}$ production calculated for adjacent measurements. Explanations as in Table 3.

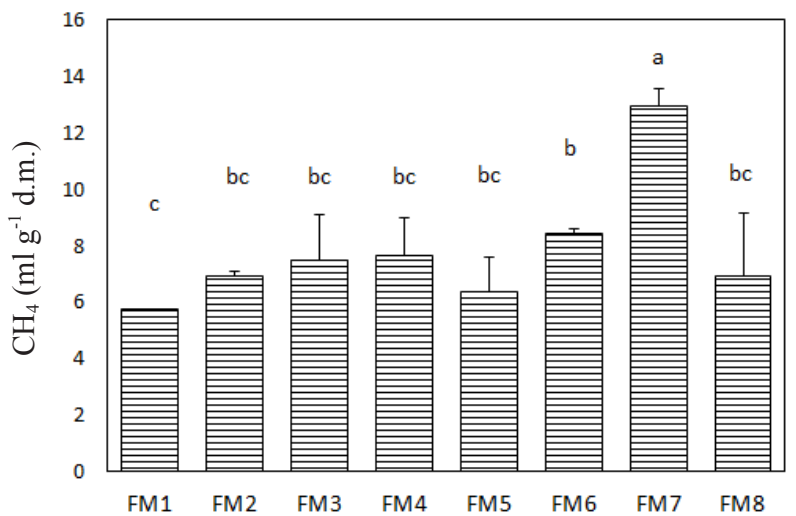

Fig. 2. Methane production rates in samples collected at various stages of anaerobic digestion. Bars with the same letter do not differ significantly at $\mathrm{p}<0.0001$, means $\pm \mathrm{SD}, n=3$. Explanations as in Table 3 .

changed and sample FM7 began intensive gas production up to $150.6 \pm 7.15 \mathrm{ml} \mathrm{g}^{-1} \mathrm{~d} . \mathrm{m}$. on day 21 and $166.9 \pm 21.4 \mathrm{ml}$ $\mathrm{g}^{-1}$ d.m. on day 35 . In turn, methane formation in sample FM8 dynamically increased after only 21 days of the incubation, with a final cumulative amount of $123.9 \pm 43.7 \mathrm{ml} \mathrm{g}^{-1}$ d.m. In the other samples, methane slowly increased until the end of the incubation and reached approximately 100$150 \mathrm{ml} \mathrm{CH}_{4} \mathrm{~g}^{-1}$ d.m.

The daily changes in the methane production presented in the inset in Fig. 1 clearly illustrate the differences among the tested samples. Methanogenic activity was activated earlier in samples FM1-FM6 (with a peak on day 14) compared with that of sample FM7 (maximum on day 21) and particularly in FM8 (maximum only on day 30). Sample FM8 was the last collected in the bioreactor after a waste retention time of 61 days. The maximum daily production of methane in this sample was relatively low; however, the final amount of accumulated methane was in the middle of the range observed for all the other samples.
Differences in the total amounts of methane accumulated by the samples during the entire incubation as well as the daily changes in gas production (Fig. 1) were not significant, as demonstrated by the ANOVA test ( $p>0.05)$. In turn, the methanogenic activity significantly $(p<0.0001)$ differed among the tested samples when the rates were calculated from the slopes of the cumulative curves (Fig. 2). The linear parts of the cumulative curves were used for the calculations, which ensured that neither adaptation nor significant growth of biomass occurred during the test period and, therefore, allowed the methanogenic activity to be related to the microbial population in the sample. As expected, the methanogenic activity was the highest in sample FM7 $\left(12.96 \pm 0.623 \mathrm{ml} \mathrm{CH}_{4} \mathrm{~g}^{-1}\right.$ d.m. $\left.\mathrm{d}^{-1}\right)$ despite the fact that it was activated slightly later than the others. The lowest activity was found for sample FM1 $(5.76 \pm 0.019 \mathrm{ml}$ $\mathrm{CH}_{4} \mathrm{~g}^{-1} \mathrm{~d} . \mathrm{m} . \mathrm{d}^{-1}$ ); however, the initiation of methane formation began earlier than in the other samples. Community dynamics was synchronised over long periods, which was 
in agreement with the findings of Brzezińska et al. (2012) and Vanwonterghem et al. (2014), who suggested niche specialization and the major role of deterministic processes within a highly controlled anaerobic system. The anaerobic digestion of organic wastes enables management thereof as well as energy and nutrient recovery. Methane formation in the tested samples was entirely based on residual organic carbon that was still present in the collected samples of the digested substrates. Such a procedure allowed the methanogenic activity to be compared at different stages of the bioreactor operation under various retention times. In our experiment, no extra organic carbon was added. Therefore, the measured activity did not express the potential methanogenic activity of the wastes, also known as the ultimate methane productivity or the biochemical methane potential (Vedrenne et al., 2008), which is typically measured with an excess substrate. More likely, the methane that formed can be assumed to be an index of the methanogenic activity of the methanogenic population developed at particular stages of the feedstock biodegradation when the samples were collected.

The methanogenic activity differed among the samples. The highest methane yield was obtained for sample FM7 (166.9 ml CH $\mathrm{g}^{-1}$ d.m., corresponding to $4.88 \mathrm{mmol}$ $\mathrm{CH}_{4} \mathrm{~g}^{-1}$ d.m.). This result is in agreement with the highest methane production during methanogenesis under a waste retention time of 47 days and confirmed the results concerning the microbial community structure (t-RFLP, NGS), which indicated that the acetotrophic Methanosaeta genus was the most active and important during the methanogenic stage. Esposito et al. (2012) observed a methane potential of approximately 7-8 $\mathrm{mmol} \mathrm{CH}_{4} \mathrm{~g}^{-1} \mathrm{VS}$ for different organic wastes, and Shah et al. (2014) reported that the average efficiency of methanogenesis reached approximately $0.24 \mathrm{~m}^{3}$ of methane from $1 \mathrm{~kg}$ of dry organic matter (i.e. $240 \mathrm{ml} \mathrm{g}^{-1}$ d.m.). This result indicates that a large amount of biodegradable organic matter is retained in the digested substrate after digestion is completed, because methanogenic activity can reach more than half of the reported results, even if the samples are not amended with a fresh substrate.

Total DNA was extracted from eight digested samples collected from an anaerobic bioreactor during various stages of anaerobic digestion (under different waste retention times), and a DNA template of each sample was used to generate amplicons of the mcrA gene ( $\sim 500 \mathrm{bp})$. Methanogenic Archaea were detected by PCR of the $m c r A$ gene in all of the tested digested samples (FM1 to FM8). T-RFLP-mcrA analysis was used to assess the diversity and the structure of the methanogenic communities. The DNAbased t-RFLP fingerprinting patterns of the methanogenic community obtained from the eight stages of anaerobic digestion are shown in Fig. 3.

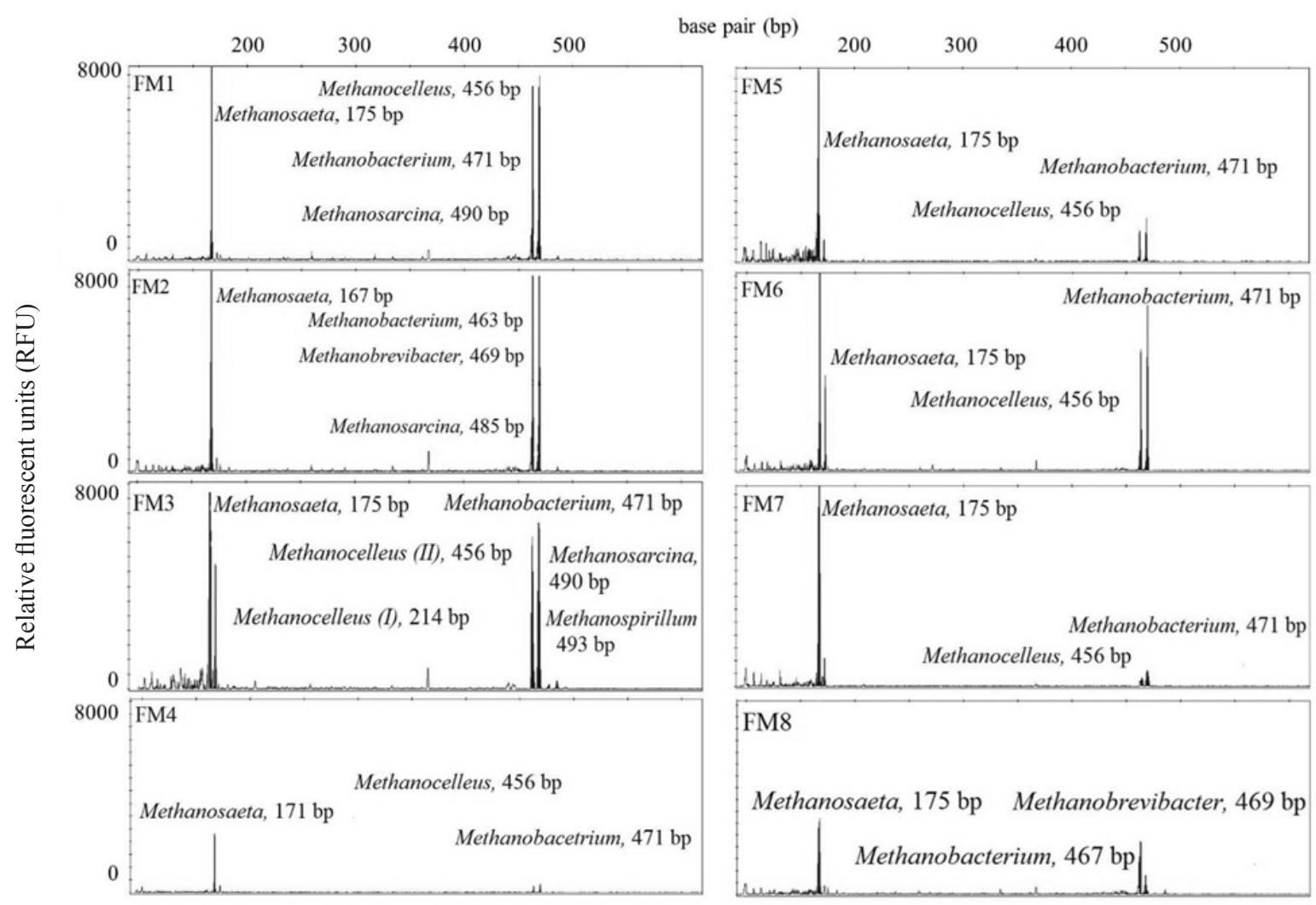

Fig. 3. t-RFLP patterns based on the mcrA gene analysis. The picture presents differences or similarities between DNA extracted from microorganisms present in the anaerobic digester. Explanations as in Table 3. 
T a b l e 2. Measured and predicted T-RF lengths (bp). The T-RF length was defined on based restriction morA genes endonucleases HaeIII

\begin{tabular}{lcc}
\hline & \multicolumn{2}{c}{ HaeIII } \\
\cline { 2 - 3 } Organism & \multicolumn{2}{c}{ T-RF (bp) } \\
\cline { 2 - 3 } & Predicted* & Measured \\
\hline Methanosaeta & 175 & 167 \\
Methanoculleus & 214 & 208 \\
Methanobacterium & 467 & 463 \\
Methanobrevibacter & 469 & 469 \\
Methanosarcina & 490 & 485 \\
Methanospirillum & 493 & 493 \\
\hline
\end{tabular}

*Nikolausz et al. (2013).

The mcrA profiles from these environments differ from each other. This result suggests that changes occur in methanogenic archaeal communities during the anaerobic process. The results of the t-RFLP profiling of the $m c r A$ genes proved the taxonomic affiliation of the predominant methanogens (Table 2). In total, six characteristics of terminal restriction fragments (T-RFs) were detected as major peaks in electrophoresis. The detected t-RFs can be assigned to the following genera: Methanosaeta, Methanoculleus, Methanobacterium, Methanobrevibacter, Methanosarcina and Methanospirillum, which is consistent with the previous study by Nikolausz et al. (2013).
Depending on the sample, three to six genera dominated in the methanogenic community. The most commonly detected methanogens that appeared in all samples belonged to the genera, Methanosaeta, Methanobacterium and Methanobrevibacter. The peak specific for Methanosarcina (485 bp) was recorded for samples FM1, FM2 and FM3. All six peaks were detected only in FM3. Furthermore, peaks distinguishing Methanoculleus (208 bp) and Methanospirillum (493 bp) were found only in FM3. The highest methane concentration in the biogas was determined in sample FM7 (retention time of 47 days) and the lowest in FM1 (retention time of 21 days) when Methanosaeta and Methanobacterium were dominant in the sludge, respectively. This result was confirmed by the nextgeneration sequencing (NGS) described below and was in agreement with methane production of these two samples. The results showed the presence of Methanobrevibacter in digested biomass in all retention times. The inherence of Methanobrevibacter in most samples was also confirmed by NGS analysis; however, their amounts were lower than those of Methanobacterium and Methanosaeta. The frequent occurrence of Methanobrevibacter could be connected with the organic waste mixture composition because microorganisms belonging to this genus participated in the anaerobic digestion of fruit waste and are typical for such environments (Bouallagui et al., 2004). In our study, the organic substrate mixture contained $25 \%$ of fruit processing waste.

The composition of the Archaea genus in all samples is illustrated in Fig. 4. The Methanobacterium and Methanosaeta constituted the dominant genera in the library

\section{Methanosphaera $\square$ Methanosarcina Methanosaeta Methanobrevibacter $\mathbb{\mathbb { N }}$ Methanobacterium}

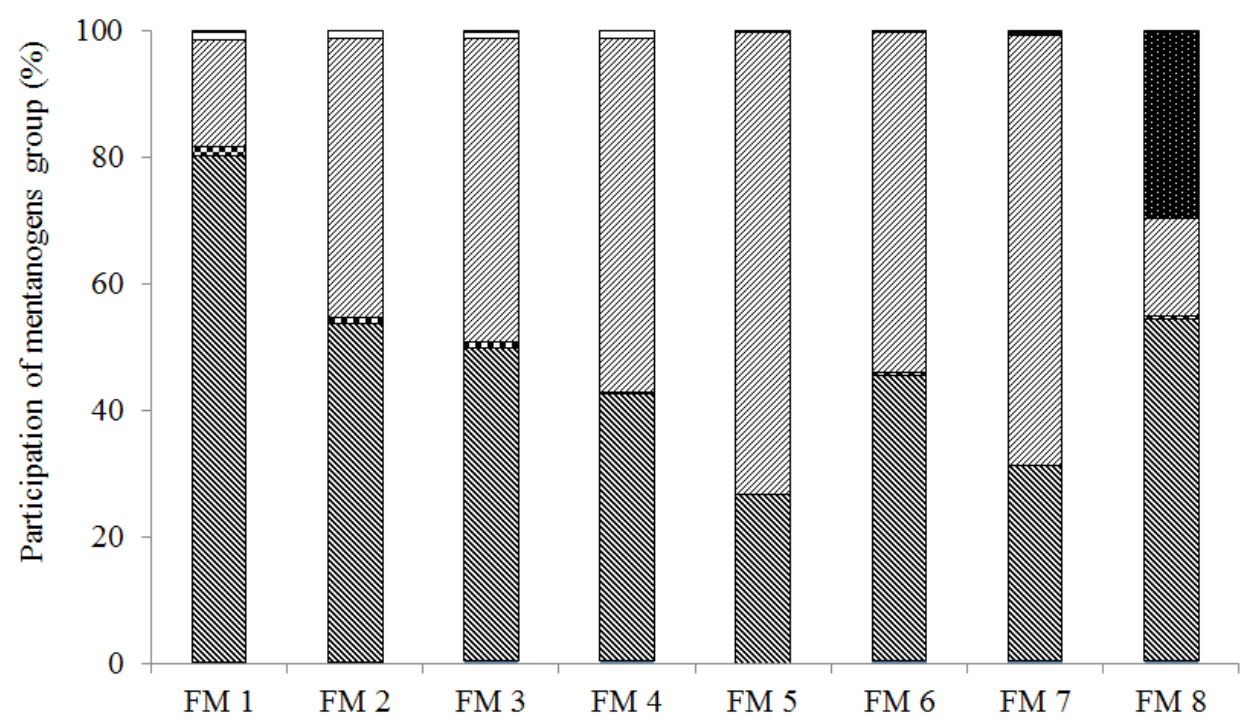

Fig. 4. Abundance of methanogen genera in particular samples detected by next-generation sequencing. Explanations as in Table 3. 


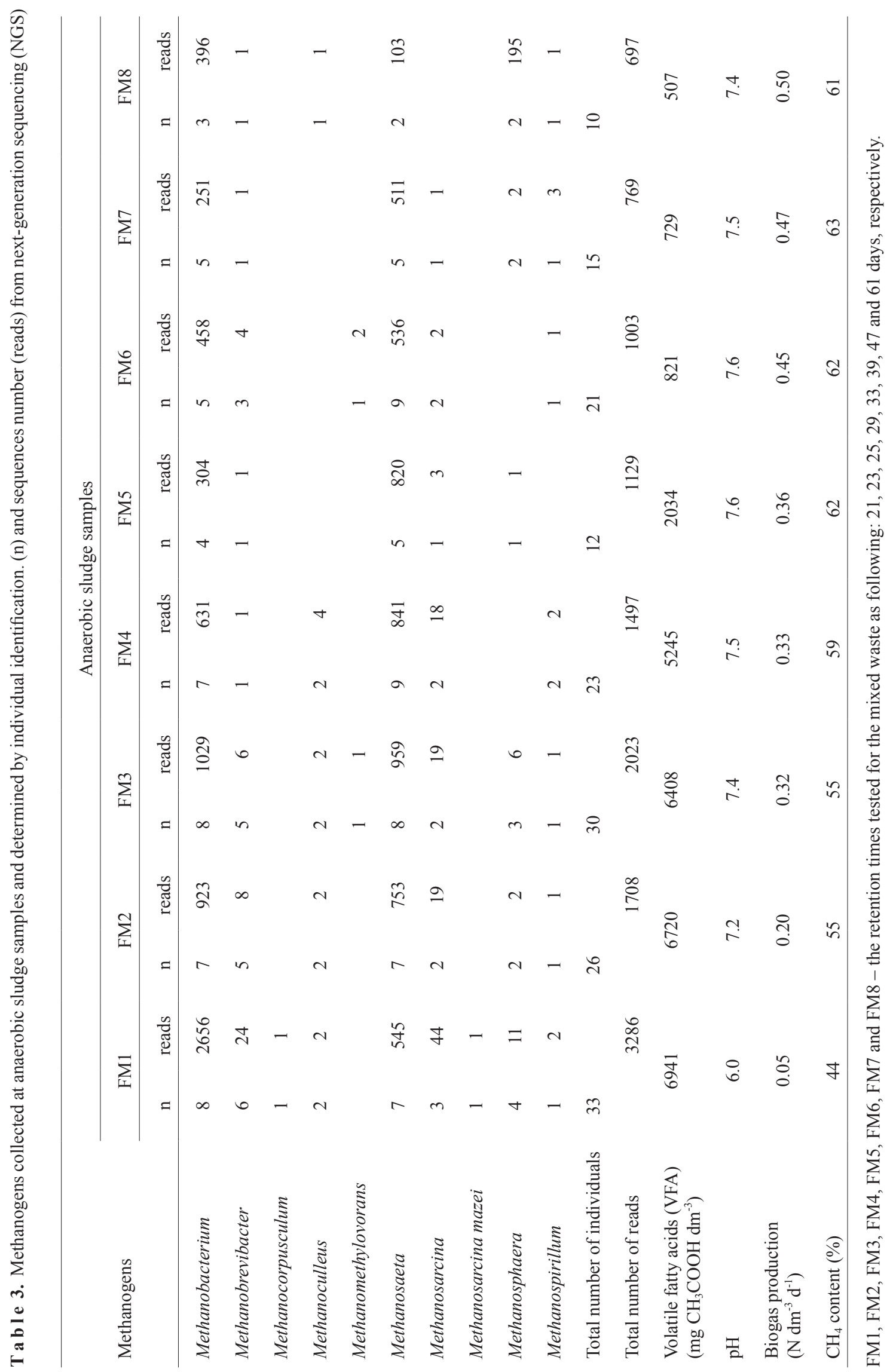


of all studied samples; however, the proportion between these microorganisms depended on the stage and retention time of the anaerobic digestion process. Methanobacterium accounted for $79.92,53.49,49.53,42.06,26.55,45.03$, 30.65 , and $54.11 \%$ of the total methanogen clones in samples FM1, FM2, FM3, FM4, FM5, FM6, FM7 and FM8, respectively, followed by Methanosaeta, which accounted for $16.74,44.01,48.00,55.95,73.08,53.53,67.94$, and $15.48 \%$, respectively. The samples (FM1- FM4) collected under the shorter waste retention times (21-29 days) had higher abundance of Methanobacterium, known as hydrogenotrophic methanogens. For these samples, the volume of biogas decreased, and the concentration of $\mathrm{CH}_{4}$ in the biogas was lower than $60 \%$ (Table 3 ). The function of hydrogenotrophic methanogens is extremely important in the anaerobic use of simple soluble compounds; however, their role in biomass processing of complex organic compounds is poorly understood (Demirel and Scherer, 2008). The samples (FM4-FM7) collected under the longer waste retention times (29-47 days), which are characterised by higher abundance of Methanosaeta, are known to utilise acetate as the only substrate for methanogenesis.

The community of methanogens in sample FM8 was dominated by Methanobacterium, although other archaeal microorganisms appeared as well (Table 3). The dominance of hydrogenotrophic Methanobacterium under the shorter waste retention times was not correlated with a high methane yield. These results indicated that if methanogenesis was performed primarily by hydrogenotrophic methanogens, the stability of the process was maintained; however, at the same time, the efficiency of biogas production and methane concentration were unsatisfactory. Methanosaeta, which appear under longer waste retention times, are known to utilise acetate as the only substrate for methanogenesis (Wang et al., 2014). At the end of the anaerobic digestion process, Methanosaeta were outcompeted by Methanosphaera, which as a hydrogenotrophic group, prefers $\mathrm{H}_{2}$ and $\mathrm{CO}_{2}$ as substrates for methane production (Bouallagui et al., 2004). These results suggest that under longer retention times, acetotrophic methanogens are the major groups involved in biogas and methane production (Fig. 1). All of these results confirm the predominance of acetotrophic Methanosaeta in the methanogenic stage and indicate the highest involvement of hydrogenotrophs Methanobacterium in the biogas production at the beginning of methanogenesis. The obtained results are in agreement with Shimada et al. (2011) and Walter et al. (2012), who confirmed that hydrogenotrophs are found in the acidogenic and at the beginning of the methanogenic stage in an anaerobic digester. However, this result is different from the results of Wang et al. (2014), who showed that methanogenesis was primarily performed by acetoclastic methanogens throughout both the acidogenic and methanogenic stages.
The results revealed that the composition of the methanogen community is dependent on the retention time, type of digested waste and stage of methanogenesis and also on the conditions in the bioreactor, such as the $\mathrm{pH}$, methane production and the concentration and composition of volatile fatty acids (VFA) (Table 3). The study by Abendroth et al. (2015) confirmed the link between the digester type, chemical parameters and microbial biocenoses. The results of our study showed that, despite the high VFA concentration $\left(6941 \mathrm{mg} \mathrm{cm}^{-3}\right)$, no decrease in the methanogen population was observed. It can be inferred that the high concentration of VFA stimulated these microorganisms. The content of VFA decreased with the extended retention time (Table 3).

The diversity of microbial communities in the bioreactor differed for the various retention times. The results suggest that Methanosaeta, Methanobacterium and Methanobrevibacter were the most commonly detected methanogens. Theincrease in the relative abundance of Methanosaeta corresponded with the decrease in Methanobacterium in the bioreactor. The presence of Methanosaeta changed from sample to sample, which predominated at the natural $\mathrm{pH}$ conditions between 7.2-7.6.

The phylogenic relationship of the representative sequences of each major OTU was used to construct a phylogenetic tree of methanogens involved in methanogenesis. As illustrated in Fig. 5, the phylogenetic tree of the $16 \mathrm{~S}$ rRNA gene sequences demonstrated that methanogens were grouped primarily into three separate clusters. This can be explained by the methanogen community structure presented in Fig. 4 and Table 3. The sequences identified in FM1 (Fig. 5), as a separated bar, belonged primarily to Methanobacterium and dominated during the short retention times; however, with a small contribution of

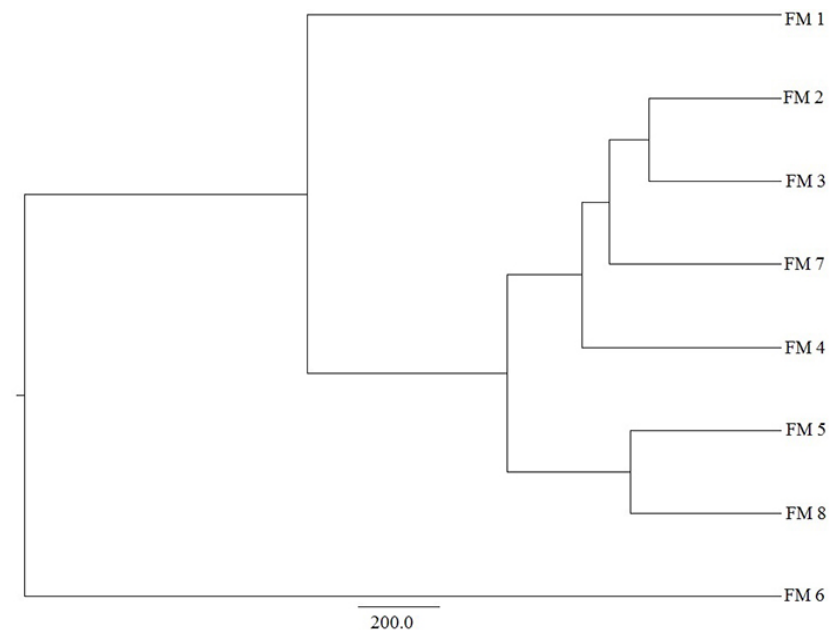

Fig. 5. Phylogenetic tree for representative $16 \mathrm{~S}$ rRNA gene sequences. The scheme indicates the phylogenetic relationships between microorganisms in various stages of anaerobic digestion. The tree was constructed by Qimme. Explanations as in Table 3. 
genera belonging to Methanosaeta, Methanobrevibacter, Methanoculleus, Methanocorpusculum, Methanosphaera, Methanospirillum, Methanosarcina and Methanosarcina mazei, the substrates varied, e.g. $\mathrm{H}_{2}, \mathrm{CO}_{2}$, acetate, methylamines and methanol. Sample FM1 was characterised by the greatest methanogen diversity among all of the studied samples. The sequences identified in FM2, FM3, FM4, FM5, FM7 and FM8 showed a high similarity between one another and were grouped in separate clusters (Fig. 5), because the sequences from these clusters are greatly related to the methanogenic Archaea belonging to acetotrophic or hydrogenotrophic microorganisms. The primary characteristic of cluster FM6 was the equal number of sequences with a similarity to acetotrophic and hydrogenotrophic methanogens.

The PCA confirmed the high correlation between the community composition and methane yield. Principal component analysis generated the two components. All the variables are visualised graphically in a correlation circle (Fig. 6). The arrowhead lines that intersect at the centre characterise the loadings of the variables. The first and second principal components ( $\mathrm{PC} 1$ and $\mathrm{PC} 2$ ) explained $65.50 \%$ of the total variability of the data set. PC1, which explains $47.57 \%$ of the variance, had the highest positive loading for Methanobacterium (0.977), Methanosarcina (0.954) and Methanobrevibacter (0.946) and a negative loading for methane yield (-0.764) (Table 4). PC2, which explains $17.93 \%$ of the variance, had the highest negative loading for Methanospirillum (-0.801) and a positive loading for Methanosphaera (0.671).
T a b l e 4. Loadings for each variable along PC1 and PC2 (first and second principal components, respectively) resulting from principal components analysis

\begin{tabular}{lll}
\hline Variable & PC1 & PC2 \\
\hline Methanobacterium & 0.978 & -0.116 \\
Methanobrevibacter & 0.947 & -0.117 \\
Methanocorpusculum & 0.921 & -0.125 \\
Methanoculleus & 0.447 & -0.103 \\
Methanomethylovorans & -0.266 & -0.113 \\
Methanosaeta & 0.099 & -0.314 \\
Methanosarcina & 0.954 & -0.196 \\
Methanosarcina mazei & 0.921 & -0.125 \\
Methanosphaera & -0.140 & 0.671 \\
Methanospirillum & 0.106 & -0.802 \\
Methane production rate & -0.595 & -0.701 \\
Methane yield & -0.765 & -0.590 \\
\hline
\end{tabular}

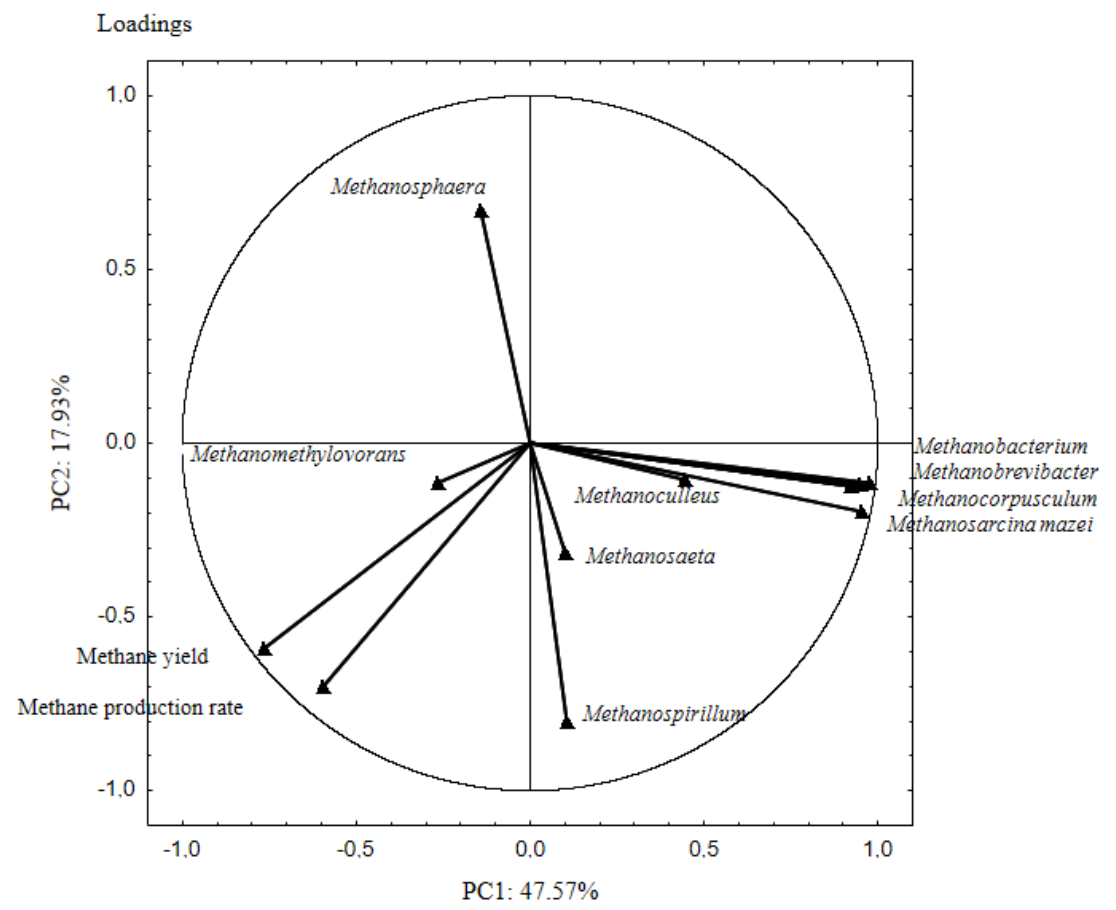

Fig. 6. Microbial community in correlation to methane yield. Principal component analysis (PCA loadings) on variable data from all studied samples. Each line represents the trajectory in the PCA plot based on the community structure and methanogenic activity of all studied samples. 


\section{CONCLUSIONS}

1. The results of this study demonstrate that the stages of the anaerobic digestion process and waste retention times determine the microbial composition.

2. The microbial community composition of an organic waste mixture corresponded with the methanogenic activity of the tested samples. Methanogenesis was primarily performed by acetotrophic methanogens (Methanosaeta) during the methanogenic stage and hydrogenotrophs (Methanobacterium) involved in the biogas production. The proportions of methanogen genera in digested samples caused changes in methanogenic activity and methane production. The most stable, dominant, and acclimated microbial communities in all samples belonged to the genera Methanosaeta and Methanobacterium. These results also increase the knowledge regarding stable microbial core biocenosis - Archaea, adapted to each retention time conditions.

3. The concentration of volatile fatty acids of about $7000 \mathrm{mg} \mathrm{dm}^{-3}$ was not an inhibitory concentration for methanogen growth, and it can be concluded that such a high concentration caused an increase in their population.

4. The results provide a dataset of Archaea present in an anaerobic digester under different retention times, which could be used for future diagnostic strategies to predict biogas production based on the microbial composition.

\section{ACKNOWLEDGEMENT}

Authors contribution: FM, ZK, BM conceived the study, participated in its design and coordination, and helped to draft the manuscript. GA, FM, OK, BWN carried out the molecular genetic studies, participated in the sequence alignment and drafted the manuscript. GA, BM, FM performed the statistical analysis. All authors wrote, read, and approved the final version of the manuscript.

Conflict of interest: The authors declare no conflict of interest.

\section{REFERENCES}

Abendroth C., Vilanova C., Günther T., Luschnig O., and Porcar M., 2015. Eubacteria and archaea communities in seven mesophile anaerobic digester plants in Germany. Biotechnol. Biofuels, 1, 8-87.

Angelidaki I. and Sanders W., 2004. Assessment of the anaerobic biodegradability of mactopollutants. Reviews in Environ. Sci. Biotechnol., 173, 117-129.

APHA (American Public Health Association), 1998. Standard methods for the examination of water and wastewater. APHA, Washington, DC, USA.

Bouallagui H., Torrijos M., Godon J.J., Moletta R., Cheikh R.B., and Touhami Y., 2004. Microbial monitoring by molecular tools of a two-phase anaerobic bioreactor treating fruit and vegetable wastes. Biotechnology Letters, 10, 857-862.

Brzezińska M., Nosalewicz M., Pasztelan M., and Włodarczyk T., 2012. Methane production and consumption in loess soil at different slope position. Scientific World J., 1-8.
Caporaso J.G., Lauber C.L., Walters W.A., Berg-Lyons D., Huntley J., Fierer N., Owens S.M., Betley J., Fraser L., Bauer M., Gormley N., Gilbert J.A., Smith G., and Knight R., 2012. Ultra-high-throughput microbial community analysis on the Illumina HiSeq and MiSeq platforms. ISME J., 6, 1621-1624.

Demirel B. and Scherer P., 2008. The roles of acetotrophic and hydrogenotrophic methanogens during anaerobic conversion of biomass to methane: a review. Reviews in Environ. Sci. Biotechnol., 7, 173-190.

DeSantis T.Z., Hugenholtz P., Larsen N., Rojas M., Brodie E.L., Keller K., Huber T., Delevi D., Hu P., and Andersen G.L., 2006. Greengenes, a chimera-checked $16 \mathrm{~S}$ rRNA gene gatabase and workbench compatible with ARB. App. Environ. Microbiol., 72, 5069-5072.

Enitan A.M., Kumari S., Swalaha F.M. Adeyemo J., Ramdhani N., and Bux F., 2014. Kinetic modeling and characterization of microbial community present in a full-scale UASB reactor treating brewery effluent. Microbial Ecology, 62, 358-368.

Esposito G., Frunzo L., Liotta F., Panico A., and Pirozzi F., 2012. Bio-methane potential test to measure the biogas production from the digestion and co-digestion of complex organic substrates. Open Environmental Eng. J., 5, 1-8.

Frąc M. and Ziemiński K., 2012. Methane fermentation process for utilization of organic waste. Int. Agrophys., 26, 317-330.

Garcia J.L., Patel B.K., and Ollivier B., 2000. Taxonomic phylogenetic and ecological diversity of methanogenic Archaea. Anaerobe, 6, 205-226.

Hansen T.L., Schmidt J.E., Angelidaki I., Marca E., Jansen J., Mosbaek H., and Christensen T.H., 2004. Method for determination of methane potentials of solid organic waste. Waste Management, 24, 393-400.

Kane E.S., Chivers M.R., Turetsky M.R., Treat C.C., Petersen D.G., Waldrop M., Harden J.W., and McGuire A.D., 2013. Response of anaerobic carbon cycling to water table manipulation in an Alaskan rich fen. Soil Biol. Biochemistry, 85, 50-60.

Kitts C.L., 2001. Terminal restriction fragment patterns: a tool for comparing microbial communities and assessing community dynamics. Current Issues Intestinal Microbiol., 2, 7-25.

Lipiec J., Brzezińska M., Turski M., Szarlip P., and Frąc M., 2015. Wettability and biogeochemical properties of the drilosphere and casts of endogeic earthworms in pear orchard. Soil Till. Res., 145, 55-61.

Nikolausz M., Walter R.F., Sträuber H., Liebetrau J., Schmidt T., Kleinsteuber S., Bratfisch F., Günther U., and Richnow H.H., 2013. Evaluation of stable isotope fingerprinting techniques for the assessment of the predominant methanogenic pathways in anaerobic digesters. App. Microbiol. Biotechnol., 5, 2251-2262.

Pervin H.M., Dennis P.G., Lim H.J., Tyson G.W., Batstone D.J., and Bond P.L., 2013. Drivers of microbial community composition in mesophilic and thermophilic temperaturephased anaerobic digestion pre-treatment reactors. Water Res., 47, 7098-7108.

Raposo F., de la Rubia M.A., Borja R., and Alaiz M., 2008. Assesment of a modiefied and optimised method for determining chemical oxygen demand of solid substrates and solutions with high suspended solid content. Talanta, 76, 448-453. 
Shah F.A., Mahmood Q., Shah M.M., Pervez A., and Asad S.A., 2014. Microbial ecology of anaerobic digesters: The key players of anaerobiosis. Scientific World J., 24, 1-21.

Shimada T., Morgenroth E., Tandukar M., Pavlostathis S.G., Smith A., Raskin L., and Kilian R.E., 2011. Syntrophic acetate oxidation in two-phase (acid-methane) anaerobic digesters. Water Sci. Technol., 2, 812-1820.

Song L., Wang, Y., Tang W., and Lei Y., 2015. Archaeal community diversity in municipal waste landfill sites. Appl. Microbiol. Biotechnol., 14, 6125-37.

Steinberg L.M. and Regan J.M., 2008. Phylogenetic comparison of the methanogenic communities from an acidic, oligotrophic fen and an anaerobic digester treating municipal wastewater sludge. Appl. Environ. Microbiology, 74, 6663-6671.

Vanwonterghem I., Jensen P.D., Dennis P.G., Hugenholtz P., Rabaey K., and Tyson G.W., 2014. Deterministic processes guide long-term synchronised population dynamics in replicate anaerobic digesters. ISME J., 8, 2015-2028.
Vedrenne F., Béline F., Dabert P., and Bernet N., 2008. The effect of incubation conditions on the laboratory measurement of the methane producing capacity of livestock wastes. Bioresource Technol., 99, 146-155.

Walter A., Knapp B.A., Farbmacher T., Ebner C., Insam H., and Franke-Whittle I.H., 2012. Searching for links in the biotic characteristics and abiotic parameters of nine different biogas plants. Microbial Biotechnol., 5, 717-730.

Wang C., Zuo J., Chen X., Xing W., Xing L., Li P., Lu X., and Li C., 2014. Microbial community structures in an integrated two-phase anaerobic bioreactor fed by fruit vegetable wastes and wheat straw. J. Environ. Sci., 12, 2484-2492.

Whang L.M., Hu T.H., Liu P.W., Hung Y.C., Fukushima T., Wu Y.J., and Chang S.H., 2015. Molecular analysis of methanogens involved in methanogenic degradation of tetramethylammonium hydroxide in full-scale bioreactors. App. Microbi. Biotechnol., 99, 1485-1497.

Ziemiński K., Romanowska I., Kowalska-Wentel M., and Cyran M., 2014. Effects of hydrothermal pretreatment of sugar beet pulp for methane production. Bioresource Technol., 166, 187-193. 